\title{
A contribuição do Modelo FPEIR nos estudos das bacias hidrográficas brasileiras
}

The contribution of the DPSIR Model in the studies of Brazilian watersheds

La contribución del Modelo FPEIR en los estudios de cuencas hidrográficas brasileñas

\section{Bruna A. Branchi}

Professora Doutora, PUC-Campinas, Brasil

bruna.branchi@puc-campinas.edu.br

\section{Denise Helena Lombardo Ferreira}

Professora Doutora, PUC-Campinas, Brasil lombardo@puc-campinas.edu.br 


\title{
RESUMO
}

O aumento populacional e a exploração dos recursos naturais têm colocado em risco a preservação dos recursos hídricos, de forma que algumas bacias hidrográficas brasileiras já se encontram em degradação ambiental. O modelo Força Motriz-Pressão-Estado-Impacto-Resposta é uma importante ferramenta aplicada no estudo da sustentabilidade das bacias hidrográficas. O objetivo deste estudo é investigar o perfil das pesquisas publicadas na Biblioteca Digital Brasileira de Teses e Dissertações sobre a aplicação do método Força Motriz-Pressão-Estado-Impacto-Resposta em bacias hidrográficas. Para isso é utilizada a abordagem exploratória descritiva. Os resultados mostram que das onze publicações encontradas no período de 2006 a 2019, sete são teses e quatro são dissertações, sendo que $64 \%$ são dos últimos quatro anos. Todas as publicações são de universidades públicas e, na quase totalidade, federais, com destaque as Universidades Federais do Ceará e do Espírito Santo. A área de avaliação da Capes Multidisciplinar é a mais frequente, totalizando cinco pesquisas entre teses e dissertações em Ciências Ambientais, Oceanografia Ambiental e Desenvolvimento e Meio Ambiente. Das onze publicações selecionadas, oito usam o modelo Força Motriz-Pressão-Estado-Impacto-Resposta para identificar indicadores que permitem avaliar a qualidade ambiental da bacias hidrográfica. Com exceção de uma dissertação, em todas as demais publicações prevalece a abordagem de pesquisa qualitativa.

PALAVRAS-CHAVE: Recursos Hídricos. DPSIR. Desenvolvimento Sustentável.

\begin{abstract}
The increase in population and the exploitation of natural resources have put the preservation of water at risk, with some Brazilian watersheds already environmentally degraded. The Driving Force-Pressure-State-Impact-Response model is an important tool applied in the study of river basin sustainability. This study aims to investigate the profile of the research published in the Brazilian Digital Library of Theses and Dissertations on the application of the Driving Force-Pressure-State-Impact-Response method in the Brazilian watersheds. For this, the exploratory descriptive approach is used. The results show that between the eleven publications selected, covering the years from 2006 to 2019 , seven are PhD theses and four are MSc dissertations, with $64 \%$ published in the last four years. All publications are from public universities and, almost entirely, federal ones, with a special position for the Federal Universities of Ceará and Espírito Santo. The most frequent Capes evaluation area is the Multidisciplinary field, totaling five publications, between theses and dissertations, in Environmental Sciences, Environmental Oceanography, and Development and Environment. One of the main results is that eight out of eleven studies use the Driving ForcePressure-State-Impact-Response model to identify indicators that allow assessing the environmental quality of the watersheds. Except for one dissertation, in all other publications the qualitative research approach prevails.
\end{abstract}

KEYWORDS: Water. DPSIR. Sustainable Development.

\section{RESUMEN}

El aumento de la población y la explotación de los recursos naturales han puesto en riesgo la preservación de los recursos hídricos, por lo que algunas cuencas hidrográficas brasileñas ya están en degradación ambiental. El modelo Fuerza Motriz-Presión-Estado-Impacto-Respuesta es una herramienta importante aplicada en el estudio de la sostenibilidad de la cuenca fluvial. El objetivo de este estudio es investigar el perfil de la investigación publicada en la Biblioteca Digital Brasileña de Tesis y Disertaciones sobre la aplicación del método Fuerza motriz-Presión-EstadoImpacto-Respuesta en cuencas fluviales. Para esto, se utiliza el enfoque descriptivo exploratorio. Los resultados muestran que de las once publicaciones encontradas en el período de 2006 a 2019, siete son tesis y cuatro son disertaciones, con un $64 \%$ de los últimos cuatro años. Todas las publicaciones son de universidades públicas y, casi en su totalidad, federales, con énfasis en las universidades federales de Ceará y Espírito Santo. El área de evaluación de Capes Multidisciplinar es la más frecuente, con un total de cinco investigaciones entre tesis y disertaciones en Ciencias Ambientales, Oceanografía Ambiental y Desarrollo y Medio Ambiente. De las once publicaciones seleccionadas, ocho utilizan el modelo Fuerza motriz-Presión-Estado-Impacto-Respuesta para identificar indicadores que permitan evaluar la calidad ambiental de las cuencas hidrográficas. Con la excepción de una disertación, en todas las demás publicaciones prevalece el enfoque de investigación cualitativa.

PALABRAS CLAVE: Recursos hídricos. DPSIR. Dessarollo sustentable. 


\section{Introdução}

No Brasil, é recente a preocupação com o uso sustentável e racional da água. O aumento populacional e o consumo acelerado dos recursos naturais têm colocado em risco a preservação das características físicas, químicas e biológicas do sistema aquático. Algumas bacias hidrográficas brasileiras encontram-se em degradação ambiental, comprometendo a vida humana em um futuro próximo, concordando com Sartori e Gewehr (2011) ao afirmarem que os recursos naturais se encontram em extinção devido ao elevado crescimento industrial, e essa degradação põe em risco a manutenção da vida do ser humano.

A Política Nacional brasileira de recursos hídricos, através da Lei Federal 9.433 de 1997 e da Resolução 001 de 1986 do Conselho Nacional de Meio Ambiente, instituiu as bacias hidrográficas como a unidade territorial de planejamento.

De acordo com Lacerda e Cândido (2013, p. 19):

[...] dentre seus objetivos destacam-se: a manutenção da quantidade e da qualidade dos diversos usos ao longo do tempo, o uso racional e integrado dos recursos hídricos visando à sustentabilidade e à prevenção de eventos hidrológicos críticos tanto de origem natural quanto devido a interferências antrópicas.

Os Comitês de Bacias Hidrográficas são órgãos colegiados compostos por representantes do poder executivo, dos usuários de água e da sociedade civil que, entre outras atividades, são encarregados da gestão coordenada dos recursos hídricos para atender o bem-estar social e econômico e proteger o meio ambiente.

O planejamento e a gestão de bacias hidrográficas numa visão integrada devem incluir os meios físico, biótico e antrópico. De acordo com Meadows (1998) para monitorar e direcionar ações rumo ao desenvolvimento sustentável é necessário um sistema de informações coerentes a partir do qual os indicadores são derivados.

Os indicadores representam um aspecto chave na tomada de decisão para melhoria do planejamento, na perspectiva de direcionar esforços na formulação e implantação de políticas públicas.

Oliveira, Lima e Vieira (2005) destacam que, em geral, os modelos de avaliação integrada do meio ambiente definem valores para as atividades humanas responsáveis por gerar pressões (forças motrizes); e consideram os elementos do impacto no ambiente, que exigem ações de resposta nos diferentes setores (ações políticas). Além disso, levam em conta as interações do meio ambiente com o desenvolvimento social e econômico e é bastante útil para estruturar as informações necessárias.

O modelo Pressão-Estado-Resposta (PER) ou Pressure-state-response (PSR) desenvolvido pela OECD sintetiza as relações de causalidade entre

[...] as ações humanas que pressionam o meio ambiente e afetam a qualidade e quantidade de recursos naturais ("estado"); a sociedade responde a estas mudanças através de políticas ambiental, econômica e setorial e através de mudanças na conscientização e no comportamento ("resposta da sociedade") (OECD, 2003, Anexo II, Quadro 1, tradução própria). 
O modelo, portanto, orienta na seleção de indicadores de Pressão, ou seja, daqueles que descrevem a influência da ação antrópica no meio ambiente. Tais atividades alteram a qualidade e a quantidade de recursos naturais (medidos pelos indicadores de Estado) e provocam reações que visam a limitar os efeitos das ações humanas (resumidas pelos indicadores de Resposta) (RUFINO, 2002).

Até 2006, as Nações Unidas seguiam este modelo, mas desde então deram preferência à elaboração de indicadores agrupados por temas e subtemas (BELL; MORSE, 2008). Mas a relativa simplicidade do modelo PER e sua adaptabilidade em áreas e níveis variados explicam a sua ampla utilização ainda hoje (SOARES et al., 2011).

Na mesma linha de modelos que visam identificar a interação entre sociedade e meio ambiente pode-se citar o modelo Força Motriz-Estado-Resposta elaborado pela Comissão para o Desenvolvimento Sustentável das Nações Unidas e o modelo Força Motriz-Pressão-EstadoImpacto-Resposta (FPEIR) ou Driving forces-Pressures-States-Impacts-Responses (DPSIR) proposto inicialmente pela Agência Ambiental Europeia (European Environmental Agency - EEA) e adotado, desde 2000, pelo Water Framework Directive da União Europeia com a finalidade de proteger os recursos hídricos (SOARES et al., 2011).

No método FPEIR, segundo Niemeijer e Groot (2006), vários aspectos dos desenvolvimentos sociais e econômicos são as forças motrizes que exercem pressão no meio ambiente (positiva ou negativa). Essas forças conduzem às mudanças no seu estado, que, por sua vez, conduzem aos impactos na saúde humana, nos ecossistemas e nos recursos naturais, resultando em resposta da sociedade direcionada para forças motrizes, pressões e/ou estado, ou até diretamente no impacto. Em síntese esse método orienta na seleção e classificação dos indicadores, quais:

- $\quad F$ - Indicadores de força motriz - refletem as influências do homem e das atividades humanas que, quando combinadas com as condições ambientais, provocam mudanças no meio ambiente;

- $\quad$ P - Indicadores de Pressão - descrevem as variáveis que diretamente causam (ou podem causar) problemas ambientais;

- $\quad$ E - Indicadores de Estado - mostram a qualidade, ou seja, a atual condição do ambiente;

- I - Indicadores de Impacto - descrevem os efeitos das mudanças de estado; e

- $\quad \mathrm{R}$ - Indicadores de Resposta - descrevem o esforço da sociedade para resolver os problemas, sejam eles na forma de políticas, leis, tecnologias limpas, dentre outros.

Em complemento, Zare et al. (2019) destacam que o método FPEIR é útil para apoiar o escopo e a estruturação do problema no processo de modelagem de Sistemas Dinâmicos. FPEIR representa as complexidades de um problema socioecológico em termos dos seguintes elementos:

- Forças Motrizes são forças que causam pressões no sistema.

- Pressões são tensões diretas que afetam os estados do sistema.

- Estados denotam as condições do sistema.

- Impactos refletem mudanças observáveis nas condições do sistema.

- Respostas são as respostas institucionais às mudanças no sistema. 
No modelo FPEIR as causas do problema em estudo são definidas identificando um conjunto de indicadores de três tipos: Força Motriz que sintetizam as mudanças no meio ambiente provocadas pelas atividades humanas; Pressão, ou seja, variáveis que efetivamente modificam o meio ambiente; Estado quais indicadores que descrevem a condição atual do sistema. 0 problema é identificado pelas variáveis de Impactos, que resumem as mudanças ocorridas. Enfim a solução é sintetizada através de indicadores de Resposta que descrevem as ações tomadas para resolver o problema.

Para a análise das bacias hidrográficas, foco desta pesquisa, uma das etapas do modelo FPEIR envolve a identificação das pressões e os consequentes potenciais impactos nos corpos hídricos e nos usos da água (BORJA et al. 2006) devido às atividades humanas, e originam em subsequentes mudanças no estado do ambiente que advêm destas pressões e as respostas da sociedade em relação a estas mudanças.

A partir de uma pesquisa bibliográfica das publicações disponíveis na Biblioteca Digital Brasileira de Teses e Dissertações, este artigo discorre sobre a aplicação do modelo FPEIR nos estudos de bacias hidrográficas.

A estrutura do trabalho está organizada em mais quatro seções, além desta introdutória. $\mathrm{Na}$ seção seguinte, é apresentado o objetivo da pesquisa. A terceira seção discorre sobre a metodologia adotada. A quarta apresenta os resultados da pesquisa. Na última seção, são sintetizadas as principais conclusões.

\section{Objetivo}

Este estudo tem por objetivo realizar uma pesquisa bibliográfica exploratória dos estudos acadêmicos disponíveis na Biblioteca Digital Brasileira de Teses e Dissertações sobre a aplicação do método FPEIR em bacias hidrográficas.

\section{Metodologia}

Este estudo apresenta uma pesquisa bibliográfica, com abordagem exploratória descritiva, a partir da leitura de dissertações e teses sobre a aplicação da ferramenta FPEIR em bacias hidrográficas. Para Gil (2012), a pesquisa bibliográfica apresenta a vantagem de possibilitar uma cobertura ampla dos fenômenos estudados. De acordo com Collis e Hussey (2005), a pesquisa exploratória busca conhecer com maior profundidade o assunto, de modo a torná-lo mais claro e proporcionar uma visão geral acerca do fato.

A última consulta à Biblioteca Digital Brasileira de Teses e Dissertações foi realizada em 24 de abril de 2020. A busca de teses e dissertações foi realizada usando as seguintes palavras: "DPSIR" AND "bacia" AND "hidrográfica"; "FPEIR" AND "bacia" AND "hidrográfica", sem impor nenhum outro limitador. Foram encontradas 16 publicações, das quais 11 teses e 5 dissertações no período de 2006 a 2019. Após a leitura dos textos, quatro teses e uma dissertação foram excluídas tendo em vista que a metodologia FPEIR não era aplicada em bacias hidrográficas. $\mathrm{Na}$ seguinte seção serão analisados os resultados das onze publicações remanescentes. 


\section{Resultados}

A partir da consulta realizada na Biblioteca Digital Brasileira de Teses e Dissertações, as teses e dissertações selecionadas (Quadro 1) foram inicialmente classificadas de acordo com o ano da publicação (Tabela 1), Universidade (Tabela 2) e a área do conhecimento do programa de mestrado ou doutorado dos autores (Tabela 3).

Quadro 1: Dissertações e Teses que tratam da aplicação do método DPSIR em bacias hidrográficas

\begin{tabular}{|c|c|}
\hline Referência bibliográfica & Bacia Hidrográfica \\
\hline \multicolumn{2}{|l|}{ DISSERTAÇÕES } \\
\hline $\begin{array}{l}\text { ALTOÉ, P.S. Agrotóxicos no Estado do Espírito Santo: uma análise das questões legais e dos } \\
\text { efeitos nas águas superficiais do Rio Jucu causados pelo herbicida glifosato. Dissertação } \\
\text { (Mestrado Profissional em Engenharia e Desenvolvimento Sustentável) - Universidade } \\
\text { Federal do Espírito Santo, } 2018 \text {. }\end{array}$ & Bacia do Rio Jucu \\
\hline $\begin{array}{l}\text { AVELINO, I.I.F. Estudos ambientais em Bacias Hidrográficas: A utilização do modelo Força } \\
\text { Motriz, Pressão, Estado, Impacto e Resposta. Dissertação (Mestrado em Desenvolvimento } \\
\text { e Meio Ambiente) - Universidade Federal do Ceará, Fortaleza, } 2018 .\end{array}$ & $\begin{array}{l}\text { Microbacias } \\
\text { Jaguaribe e Riacho } \\
\text { do Sangue }\end{array}$ \\
\hline $\begin{array}{l}\text { FELINTO, C.M.R. Aplicação do sistema de indicadores de sustentabilidade força motriz- } \\
\text { pressão-estado-impacto-resposta (FPEIR) para a gestão de recursos hídricos em João } \\
\text { Pessoa - PB. Dissertação (Mestrado em Engenharia Civil e Ambiental) - Universidade } \\
\text { Federal de Campina Grande, } 2016 \text {. }\end{array}$ & $\begin{array}{lcc}\text { Bacias dos } & \text { Rio } \\
\text { Paraíba e } & \text { Rio } \\
\text { Gramame } & \end{array}$ \\
\hline $\begin{array}{l}\text { LANDIM NETO, F.O. Aplicação do modelo DPSIR na bacia hidrográfica do Rio Guaribas, } \\
\text { Ceará, Brasil: subsídios para a gestão ambiental local. Dissertação (Mestrado em } \\
\text { Geografia) - Universidade Federal do Ceará, } 2013 .\end{array}$ & $\begin{array}{l}\text { Bacia do Rio } \\
\text { Guaribas }\end{array}$ \\
\hline \multicolumn{2}{|l|}{ TESES } \\
\hline $\begin{array}{l}\text { SALES, J.C.A. Metodologia de análise integrada de indicadores ambientais em bacias } \\
\text { hidrográficas. Tese (Doutorado em Ciências Ambientais) - Universidade Estadual Paulista, } \\
\text { Sorocaba, } 2019 .\end{array}$ & Bacia do Rio Una \\
\hline $\begin{array}{l}\text { TEUBNER Junior, F.J. Aportes de água e nutrientes para o sistema estuarino da Baía de } \\
\text { Vitória (ES): Subsídios para a gestão ambiental integrada. Tese (Doutorado em } \\
\text { Oceanografia Ambiental) - Universidade Federal do Espírito Santo, 2016. }\end{array}$ & 10 Bacias \\
\hline $\begin{array}{l}\text { LANDIM NETO, F.O. Aplicação de indicadores de modelo força motriz pressão, estado, } \\
\text { impacto, resposta - DPSIR: Subsídios para o planejamento e gestão de bacia hidrográfica } \\
\text { do Rio São Gonçalo- CE. Tese (Doutorado em Geografia) - Universidade Federal Do Ceará, } \\
2016 .\end{array}$ & $\begin{array}{l}\text { Bacia do Rio São } \\
\text { Gonçalo }\end{array}$ \\
\hline $\begin{array}{l}\text { GONÇALVES, M.A. Ecohidrologia e gestão integrada de recursos hídricos em uma bacia } \\
\text { lacustre costeira (Lago Nova, Linhares, ES). Tese (Doutorado em Oceanografia Ambiental) } \\
\text { - Universidade Federal do Espírito Santo, } 2015 .\end{array}$ & Bacia do lago Nova \\
\hline $\begin{array}{l}\text { CALDAS, A.L.R. Método de diagnóstico para gestão participativa de recursos hídricos: } \\
\text { Estudo de caso e modelagem conceitual com enfoque DPSIR. Tese (Doutorado em Ciências } \\
\text { Ambientais) - Universidade Federal de Goiás, } 2012 .\end{array}$ & $\begin{array}{l}\text { Sub-bacia do Rio das } \\
\text { Almas }\end{array}$ \\
\hline $\begin{array}{l}\text { SILVA, L.M. Indicadores ambientais e a gestão de bacias hidrográficas de economia agrícola: } \\
\text { diagnóstico e reflexões sobre o caso da bacia do rio Preto, noroeste de Minas Gerais. Tese } \\
\text { (Doutorado em Geografia) - Universidade Federal de Minas Gerais, } 2012 .\end{array}$ & Bacia do Rio Preto \\
\hline $\begin{array}{l}\text { AZEVEDO, J. Ferramenta para Análise de Dados Socioeconômicos e Ambientais para } \\
\text { Definição de Políticas Públicas. Estudo de Caso: Bacia Ambiental do Rio Imboassú, } \\
\text { Município de São Gonçalo/RJ. Tese (Doutorado em Geociências - Geoquímica) - } \\
\text { Universidade Federal Fluminense, 2006. }\end{array}$ & $\begin{array}{l}\text { Bacia do } \\
\text { Imboassú }\end{array}$ \\
\hline
\end{tabular}

Fonte: Elaboração própria. 
A Tabela 1 destaca o número de dissertações e teses por ano no período de interesse da pesquisa. Pode-se observar que $64 \%$ dessas publicações ocorreram nos últimos quatro anos.

Tabela 1: Número de Dissertações e Teses selecionadas por ano

\begin{tabular}{|c|c|c|c|c|c|c|c|c|c|}
\hline & 2006 & 2012 & 2013 & 2014 & 2015 & 2016 & 2018 & 2019 & Total \\
\hline Dissertações & & & 1 & & & 1 & 2 & & 4 \\
\hline Teses & 1 & 2 & & & 1 & 2 & & 1 & 7 \\
\hline
\end{tabular}

Fonte: Elaboração própria.

Ao classificar as dissertações e teses de acordo com a Universidade do programa de pósgraduação, todas elas pertencem a universidades públicas e, na quase totalidade, federais (Tabela 2), com a exceção de uma tese da Universidade Estadual Paulista. As Universidades Federais do Ceará e do Espírito Santos foram aquelas com o maior número de publicações.

Tabela 2: Número de Dissertações e Teses publicadas por Universidade

\begin{tabular}{lcc}
\hline & Dissertações & Teses \\
\hline Universidade Federal do Ceará & 2 & 1 \\
Universidade Federal de Campina Grande, Paraíba & 1 & \\
Universidade Federal do Espírito Santos & 1 & 2 \\
Universidade Federal Fluminense & & 1 \\
Universidade Federal de Goiás & & 1 \\
Universidade Federal de Minas Gerais & & 1 \\
Universidade Estadual Paulista & & 1 \\
\hline
\end{tabular}

Fonte: Elaboração própria.

A distribuição dos textos selecionados de acordo com a área de avaliação da Capes (Tabela 3 e Figura 1) permite notar que a área Multidisciplinar é a mais frequente, totalizando cinco pesquisas entre teses e dissertações em Ciências Ambientais, Oceanografia Ambiental e Desenvolvimento e Meio Ambiente. Nas Ciências Humanas a única área de interesse é Geografia com uma dissertação e duas teses. A área das Engenharias apresenta o menor destaque, com apenas duas dissertações, uma na Engenharia Civil e Ambiental e outra na Engenharia e Desenvolvimento Sustentável.

Tabela 3: Número de Dissertações e Teses publicadas por área

\begin{tabular}{lcc}
\hline & Dissertações & Teses \\
\hline Geografia & 1 & 2 \\
Ciências Ambientais & & 2 \\
Oceanografia Ambiental & & 2 \\
Geoquímica & & 1 \\
Engenharia Civil e Ambiental & 1 & \\
Engenharia e Desenvolvimento Sustentável & 1 & \\
Desenvolvimento e Meio Ambiente & 1 & \\
\hline
\end{tabular}

Fonte: Elaboração própria. 
Figura 1: Classificação de Dissertações e Teses selecionadas por área de avaliação da CAPES
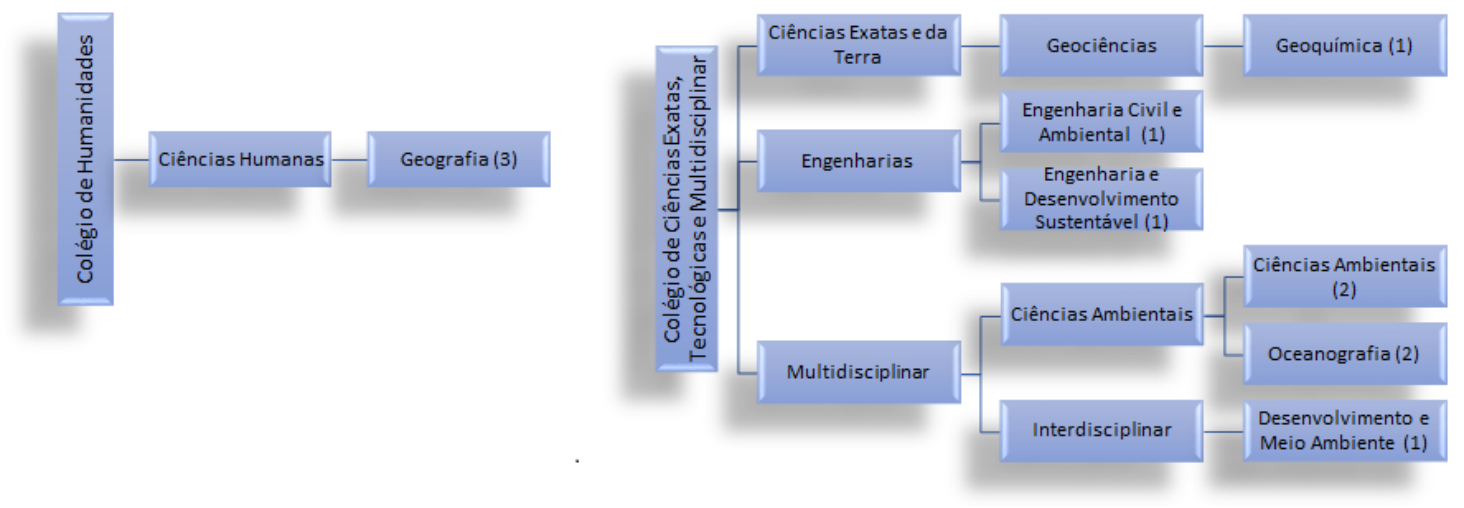

Fonte: Elaboração própria.

A revisão bibliográfica permitiu ressaltar a flexibilidade no uso do modelo FPEIR. Há autores que identificam nessa flexibilidade uma fraqueza do modelo (GARI; NEWTON; ICELY, 2015). Esta consideração pode ser apropriada se o objetivo de sua aplicação for identificar o melhor conjunto de indicadores para a avaliação de bacias hidrográficas. Entretanto, esta característica é um ponto de força de uma abordagem teórica que orienta na definição do problema e facilita a identificação das relações entre os fenômenos observados, visando sustentar a elaboração de respostas.

As diferentes aplicações não permitem identificar um conjunto de indicadores mais usados para cada parte do modelo devido aos diferentes objetivos que orientam as pesquisas.

Por outro lado, foi possível identificar alguma similaridade na aplicação do modelo quando se considera a primeira parte, ou seja, a identificação das Forças Motrizes. Geralmente são identificadas Forças Motrizes ligadas às dinâmicas demográfica, social e econômica, ou seja, derivantes da ação antrópica e que orientam a seleção dos indicadores de Pressão, Estado Impacto e Resposta.

Diferenciando-se da maioria dos estudos, Landim Neto $(2013 ; 2016)$ identifica três vetores de Força Motriz e associa a cada um deles os indicadores mais apropriados de Pressão, Estado, Impacto e Resposta. Na tese de doutoramento, estudando a bacia hidrográfica do rio São Gonçalo (CE), Landim Neto (2016) identifica as forças motrizes: a) Urbanização; b) Complexo Industrial e Portuário do Pecém; c) Exploração dos Recursos Ambientais. A mesma forças motrizes foram usadas por Landim Neto (2013) na dissertação para estudar a bacia hidrográfica do rio Guaribas (CE).

Felinto (2016) destaca uma aplicação diferente da maioria, em sua dissertação foram elaborados dois modelos FPEIR, um para o Estado "Disponibilidade hídrica superficial e subterrânea" e outro para o Estado "Qualidade da água superficial e subterrânea". Compartilhando Forças Motrizes e Pressões, os dois modelos facilitaram a identificação e análise dos diferentes Impactos e Respostas no estudo das bacias hidrográficas dos rios Paraíba e Gramame.

O modelo FPEIR foi utilizado em oito dos onze trabalhos selecionados para identificar indicadores que permitissem avaliar a qualidade ambiental da bacias hidrográfica e orientassem 
as estratégias de gestão das mesmas (SALES, 2018; AVELINO, 2018; FELINTO, 2016; LANDIM NETO, 2016 e 2013; TEUBNER JR., 2016; GONÇALVES, 2015; AZEVEDO, 2006).

Caldas (2012), diferentemente dos outros autores, usou o modelo FPEIR para complementar outras técnicas na construção de mapas conceituais. A técnica adotada foi de análise de conteúdo das entrevistas semiestruturadas aplicadas.

Silva (2012), por sua vez, utilizou o modelo FPEIR para classificar indicadores previamente selecionados a partir da técnica multicritério DELPHI.

Enfim, a única pesquisa quantitativa foi de Altoé (2018). A autora aproveita o modelo FPEIR para fundamentar a escolha de variáveis a serem usadas em modelo preditivo relacionado ao uso do glifosato e seus efeitos nas águas superficiais do rio Jucu.

Em síntese, as análises realizadas com a aplicação deste modelo foram prevalentemente do tipo qualitativo, para avaliar a qualidade ambiental da bacia hidrográfica estudada, identificar respostas apropriadas aos impactos das ações antrópicas e servir de fundamento às decisões de políticas públicas.

\section{Conclusão}

Tendo em vista a situação atual de degradação que caracteriza algumas bacias hidrográficas brasileiras e a necessidade de dispor de recursos hídricos de qualidade e em quantidade apropriada, recursos tão valiosos para a humanidade, torna-se essencial estudar indicadores que auxiliem nessa gestão. Pode-se afirmar que o modelo Força Motriz-Pressão-EstadoImpacto-Resposta (FPEIR) proposto inicialmente pela Agência Ambiental Europeia e adotado, desde 2000, pelo Water Framework Directive da União Europeia com a finalidade de proteger os recursos hídricos, é uma importante ferramenta na sistematização de indicadores de sustentabilidade, especialmente quando utilizados em bacias hidrográficas. A partir da sua aplicação é possível avaliar a qualidade ambiental da bacia hidrográfica estudada, identificar respostas apropriadas aos impactos das ações antrópicas e servir de fundamento às decisões de políticas públicas.

No estudo realizado investigou-se o perfil das pesquisas acadêmicas sobre o tema aplicação do método FPEIR em bacias hidrográficas disponíveis na Biblioteca Digital Brasileira de Teses e Dissertações.

Os resultados da pesquisa mostraram que o tema ora investigado foi estudado em sete teses e quatro dissertações no período de 2006 a 2019, sendo que 64\% dessas publicações ocorreram nos últimos quatro anos. Todas as publicações são de universidades públicas e, na quase totalidade, federais, com destaque as Universidades Federais do Ceará e do Espírito Santo, com maior número de publicações.

De acordo com área de avaliação da Capes, a área Multidisciplinar é a mais frequente, totalizando cinco pesquisas entre teses e dissertações em Ciências Ambientais, Oceanografia Ambiental e Desenvolvimento e Meio Ambiente.

O modelo FPEIR foi utilizado em oito dos onze trabalhos selecionados para identificar indicadores que permitissem avaliar a qualidade ambiental da bacias hidrográfica no sentido de orientar as estratégias de gestão dessas bacias. 
Nas publicações estudadas prevaleceu o caráter qualitativo na aplicação do modelo FPEIR, sempre com intuito de avaliar a qualidade ambiental da bacia hidrográfica estudada, identificar respostas apropriadas aos impactos das ações antrópicas e servir de fundamento às decisões de políticas públicas. Apenas uma única publicação foi encontrada, especificamente dissertação, de aplicação do modelo FPEIR com abordagem quantitativa.

\section{Referências}

ALTOÉ, P.S. Agrotóxicos no Estado do Espírito Santo: uma análise das questões legais e dos efeitos nas águas superficiais do Rio Jucu causados pelo herbicida glifosato. Dissertação (Mestrado Profissional em Engenharia e Desenvolvimento Sustentável) - Universidade Federal do Espírito Santo, 2018.

AVELINO, I.I.F. Estudos ambientais em Bacias Hidrográficas: A utilização do modelo Força Motriz, Pressão, Estado, Impacto e Resposta. Dissertação (Mestrado em Desenvolvimento e Meio Ambiente) - Universidade Federal do Ceará, Fortaleza, 2018.

AZEVEDO, J. Ferramenta para Análise de Dados Socioeconômicos e Ambientais para Definição de Políticas Públicas. Estudo de Caso: Bacia Ambiental do Rio Imboassú, Município de São Gonçalo/RJ. Tese (Doutorado em Geociências - Geoquímica) - Universidade Federal Fluminense, 2006.

BELL, S.; MORSE, S. Sustainability indicators: Measuring the unmeasurable? 2a ed. London: Earthscan, 2008.

BORJA, A. et al. The European Water Framework Directive and the DPSIR, a methodological approach to assess the risk of failing to achieve good ecological status. Estuarine - Coastal and Shelf Science, v. 66, n. 1-2, p. 84-96, 2006.

CALDAS, A.L.R. Método de diagnóstico para gestão participativa de recursos hídricos: Estudo de caso e modelagem conceitual com enfoque DPSIR. Tese (Doutorado em Ciências Ambientais) - Universidade Federal de Goiás, 2012.

COLLIS, J.; HUSSEY, R. Pesquisa em Administração: um guia prático para alunos de graduação e pós-graduação. 2. ed. Porto Alegre: Bookman, 2005.

FELINTO, C.M.R. Aplicação do sistema de indicadores de sustentabilidade força motriz-pressão-estado-impactoresposta (FPEIR) para a gestão de recursos hídricos em João Pessoa - PB. Dissertação (Mestrado em Engenharia Civil e Ambiental) - Universidade Federal de Campina Grande, 2016.

GARI, S.R.; NEWTON, A.; ICELY, J.D. A review of the application and evolution of the DPSIR framework with an emphasis on coastal social-ecological systems. Ocean \& Coastal Management, v. 103, p.63-77, jan. 2015.

GIL, A.C. Métodos e técnicas de pesquisa social. 6. ed. São Paulo: Atlas, 2012.

GONÇALVES, M.A. Ecohidrologia e gestão integrada de recursos hídricos em uma bacia lacustre costeira (Lago Nova, Linhares, ES). Tese (Doutorado em Oceanografia Ambiental) - Universidade Federal do Espírito Santo, 2015.

LACERDA, C.S.; CÂNDIDO, G.A. Modelos de indicadores de sustentabilidade para gestão de recursos hídricos. Em LIRA, W.S.; CÂNDIDO, G.A. (orgs.) Gestão sustentável dos recursos naturais: uma abordagem participativa[online]. Campinas Grande: EDUPB, 2013. Disponível em: http://books.scielo.org. Acesso em: 25 out. 2019.

LANDIM NETO, F.O. Aplicação do modelo DPSIR na bacia hidrográfica do Rio Guaribas, Ceará, Brasil: subsídios para a gestão ambiental local. Dissertação (Mestrado em Geografia) - Universidade Federal do Ceará, 2013.

LANDIM NETO, F.O. Aplicação de indicadores de modelo força motriz pressão, estado, impacto, resposta - DPSIR: Subsídios para o planejamento e gestão de bacia hidrográfica do Rio São Gonçalo- CE. Tese (Doutorado em Geografia) - Universidade Federal Do Ceará, 2016.

MEADOWS, D. Indicators and Information Systems for Sustainable: A Report to the Balaton Group. The Sustainable Institute, $1998 . \quad$ Disponível em: <http://www.biomimicryguild.com/alumni/documents/download/Indicators_and_information_systems_for_sustai nable_develoment.pdf>. Acesso em: 15 jun. 2018.

OECD. OECD environmental indicators: development, measurement and use. Reference Paper. Paris, 2003. Disponível em: <http://www.oecd.org/environment/indicators-modelling-outlooks/24993546.pdf>. Acesso em: 13 nov. 2019. 
NIEMEIJER, D.; GROOT, R. S. Framing environmental indicators: moving from causal chains to causal networks. Environment, Development and Sustainability, v. 10, n. 1, p. 89-106, 2006.

OLIVEIRA, R. E. S.; LIMA, M. M. C. L.; VIEIRA, J. M. P. An indicator system for surface water quality in river basins. Proceedings of the Fourth InterCeltic Colloquium on Hydrology and Management of Water Resources, Guimarães, Portugal, p. 128-138, 2005. Disponível em: <https://iahs.info/uploads/dms/13797.19-128-138-08-IC307-ManuelaLima.pdf> Acesso em: 6 out. 2019.

RUFINO, R. C. Avaliação da qualidade ambiental do Município de Tubarão (SC) através do uso de indicadores ambientais. 2002. 123 fls. Dissertação (Mestrado em Engenharia de Produção) - Universidade Federal de Santa Catarina. Florianópolis, SC: UFSC, 2002.

SALES, J.C.A. Metodologia de análise integrada de indicadores ambientais em bacias hidrográficas. Tese (Doutorado em Ciências Ambientais) - Universidade Estadual Paulista, Sorocaba, 2019.

SARTORI, L. P.; GEWEHR, L. O Crescimento Econômico e as Consequências das Externalidades Ambientais Negativas Decorrente do Processo Produtivo. Revista de Direito e Socioambiental, v. 2, n. 2, p. 439-458, 2011.

SOARES, A.B.; SILVA FILHO, J.C.L.; ABREU, M.C.S.; SOARES, F.de A. Revisando a estruturação do Modelo DPSIR como Base para um Sistema de Apoio à Decisão para a Sustentabilidade de Bacias Hidrográficas. Revista em Agronegócios e Meio Ambiente, v. 4, n. 3, p. 521-545, set./dez., 2011. Disponível em: <https://periodicos.unicesumar.edu.br/index.php/rama/article/view/1922/1300>. Acesso em: 14 nov. 2019.

SILVA, L.M. Indicadores ambientais e a gestão de bacias hidrográficas de economia agrícola: diagnóstico e reflexões sobre o caso da bacia do rio Preto, noroeste de Minas Gerais. Tese (Doutorado em Geografia) Universidade Federal de Minas Gerais, 2012.

TEUBNER Junior, F.J. Aportes de água e nutrientes para o sistema estuarino da Baía de Vitória (ES): Subsídios para a gestão ambiental integrada. Tese (Doutorado em Oceanografia Ambiental) - Universidade Federal do Espírito Santo, 2016.

ZARE, F. et al. Improved integrated water resource modelling by combining DPSIR and system dynamics conceptual modelling techniques. Journal of Environmental Management, v. 246, p. 27-41, 2019. 